\title{
Marked transfection enhancement by the DPL (DNA/peptide/lipid) complex
}

\author{
IK-JAE MOON ${ }^{1}$, HYUNGU KANG ${ }^{1}$, YOUNG-BAE SEU ${ }^{2}$, BYEONG-CHURL CHANG ${ }^{3}$, \\ DAE-KYU SONG ${ }^{4}$ and JONG-GU PARK ${ }^{1,3}$
}

\begin{abstract}
${ }^{1}$ WelGENE Inc., 716-3 Hosandong, Dalseogu, Daegu 704-230; ${ }^{2}$ Department of Microbiology, College of Natural Sciences, Kyungpook National University, 1370 Sangyeokdong, Bookgu, 702-701 Daegu; ${ }^{3}$ Department of Medical Genetic

Engineering, Keimyung University School of Medicine, Dongsan Medical Center, 194 Dongsandong, Joonggu,

700-712 Daegu; ${ }^{4}$ Department of Physiology, Keimyung University School of Medicine,

Dongsan Medical Center, 194 Dongsandong, Joonggu, 700-712 Daegu, Korea
\end{abstract}

Received March 6, 2007; Accepted April 10, 2007

\begin{abstract}
A short peptide, corresponding to the nuclear localization signal of the human immunodeficiency virus-1 Tat protein, Arg-Lys-Lys-Arg-Arg-Gln-Arg-Arg-Arg, was modified by adding a cysteine residue at the $\mathrm{COOH}$ terminus. The peptide was mixed with a reporter plasmid, and then with cationic lipids, to form a tripartite complex, DNA/ peptide/lipid (DPL). Various cell lines were treated with the DPL complex and compared for transfection efficiency with those of the conventional DNA/lipid (DL) complex. With the simple inclusion of the peptide, the DPL complex showed much enhanced transfection. Meanwhile, the plasmid DNA mixed only with the peptide exhibited some improvement but with much lower transfection than the DPL complex. When the DPL complex was formed with various cationic lipids, the DOSPA/DOPE exhibited superior transfection efficiency than the other cationic lipids tested at the optimal ratio of 1:3:5 (w:w:w) in many cell types. At the optimal ratio of the DPL components, transfection efficiency was routinely shown to be $\sim 10$-fold higher for reporter gene expression
\end{abstract}

Correspondence to: Dr Jong-Gu Park, WelGENE Inc., 716-3 Hosandong, Dalseogu, Daegu 704-230, Korea

E-mail: jonggu@kmu.ac.kr

Abbreviations: HIV, human immunodeficiency virus; NLS, nuclear localization signal; HPLC, high performance liquid chromatography; DOSPA, 2,3-dioleyloxy- $N$-[2( sperminecarboxamido)ethyl]- $N, N$ dimethyl-1-propan-aminium; DOPE, dioleoyl phosphatidylethanolamine; DOTMA, N-[2,3-(dioleyloxy) propyl]-N,N,Ntrimethylammonium chloride; DOTAP, dioleoyl-trimethylammoniumpropane; FBS, fetal bovine serum

Key words: lipid complex, cysteine modification, Tat peptide, cationic lipids, gene delivery, transfection, k-ras antisense than that of the conventional DL complex. Furthermore, when subcutaneous tumors of a colon cancer cell line (SW480) were treated intratumorally with antisense oligos, k-ras-RiAS, delivered as a DPL complex, tumor growth was markedly suppressed. This study shows that the DPL complex, which is easy to formulate by ordered mixing, can be employed for a much enhanced cellular uptake of a transgene both in vitro and in vivo.

\section{Introduction}

As the fields of molecular biology and gene therapy progress, an urgent need for the efficient delivery of nucleic acids into cells or tissues has emerged (1-4). To be useful for both in vitro and in vivo gene delivery, a nucleic acid must be delivered into the target cells or tissue in an effective amount. At the same time, it is desired that the nucleic acid delivery does not induce cellular cytotoxicity and host immune responses.

Gene deliveries may be performed by viral and non-viral vectors (5). For viral vectors, many different agents, such as retrovirus, adenovirus and adeno-associated virus vectors, have been employed. However, viral vectors have several drawbacks, including host immune responses to viral proteins and difficulties in the large-scale production of recombinant viruses $(6,7)$. For non-viral vectors, many different agents or methods, such as cationic lipids, polymers, calcium phosphate, electroporation and microinjection, have been used (8-12). Non-viral vectors are easy to prepare and induce less immune responses, which allow repeated administration of the vectors to the host. For these reasons, gene delivery using non-viral vectors has increasingly become popular (1). However, nonviral vectors show, in general, less transfection efficiency than those of viral vectors, necessitating further improvement.

Cationic lipids have been widely employed for gene delivery both in vitro and in vivo. In order to take advantage of the negatively charged nature of DNA and the cell surface, a large number of lipids are cationic or positively charged. Cationic lipids, complexed with nucleic acids or DNA, interact electrostatically with the cell surface, and endocytose into the cell cytoplasm. Cationic lipids mediate effective gene 
delivery into cells, but still exhibit much poorer delivery than viral vectors. Further, the efficiency of gene delivery using cationic lipids is affected by the size of the nucleic acids and host cells used. In addition, cationic lipids can induce cytotoxicity in cells, and may exhibit decreased transfection in the presence of serum $(2,3,13)$.

Previously, peptides consisting largely of basic amino acids have been shown to mediate intracellular delivery of macromolecules, including proteins and nucleic acid molecules $(14,15)$. These peptides are derived from the HIV Tat protein (16-19), SV40 large T antigen (20,21), Drosophila Antennapedia $(22)$, protamine sulfate $(23,24)$ and histone $\mathrm{H} 1$ $(25,26)$. The Tat peptide of the NLS signal sequence has been shown to improve the cellular uptake of macromolecules when chemically conjugated to, or expressed as a part of, recombinant proteins.

In the present study, the enhanced cellular uptake of plasmid DNA has been studied by forming a tripartite transfection complex of DNA/Tat peptide/cationic lipid (DPL). The transfection efficiency was investigated with various peptides of a similar nature and with the Tat peptide with C-terminal modifications (Tat-C). The mixing order and differing ratios of the three components were examined for an optimal delivery. Furthermore, the cellular uptake of antisense oligos in DPL was studied in vivo for enhanced silencing of a target gene.

\section{Materials and methods}

Cell lines and cell culture. Nine cancer cell lines, K562 (chronic myelogenous leukemia), HeLa (cervix adenocarcinoma), HepG2 (hepatoblastoma), Hep3B (liver carcinoma), A549 (lung carcinoma), HT-29 (colon adenocarcinoma), NCI-H1299 (lung carcinoma), MCF-7 (breast carcinoma) and SW480 (colon cancer) were obtained from the Korean Cell Line Bank (Seoul, Korea). Cells were cultured in either RPMI-1640 or EMEM, supplemented with $10 \%$ heat-inactivated FBS, 2 mM L-glutamine, 100 units $/ \mathrm{ml}$ penicillin and $100 \mu \mathrm{g} / \mathrm{ml}$ streptomycin. Cells were incubated in an atmosphere of $5 \% \mathrm{CO}_{2}$ at $37^{\circ} \mathrm{C}$. The cell culture reagents, including FBS, were purchased from WelGENE (Daegu, Korea). Cells were maintained at a proper density and replaced with fresh medium one day before transfection. Cell survival was examined with $0.4 \%$ trypan blue staining.

Construction of plasmid vectors with reporter genes. Transfection efficiency was examined for the expression level of reporter genes in eukaryotic expression vectors. The three reporter genes used were those encoding luciferase, $\beta$ galactosidase and GFP (Green fluorescence protein). The luciferase gene was rescued from the pGEM-luc vector (Promega, Madison, WI) and cloned into the BamHI-XhoI site of the pcDNA3 vector (Invitrogen, Carlsbad, CA), which was named pcDNA3-luc. The $\beta$-galactosidase (LacZ) gene from the pHook-2 LacZ (Invitrogen) vector was cloned into the HindIII-BamHI sites of pcDNA3, and designated pcDNA3-LacZ. The GFP gene from pEGFP-N1 (Clontech Laboratories Inc, Palo Alto, CA) was cloned into the $\mathrm{p} \Delta \mathrm{E} 1 \mathrm{sp} 1 \mathrm{~A}$ vector (Microbix Biosystems Inc., Ontario, Canada).

Peptide synthesis and modifications. The peptide used in the present study was derived from the Tat protein of HIV-1. The Tat peptide corresponds to the nuclear localization signal (NLS) sequence of 9 amino acids (49-57; Arg-Lys-Lys-ArgArg-Gln-Arg-Arg-Arg). The peptide was modified at either the $\mathrm{N}$ - or C-terminus by the addition of an amino acid (Table I). Peptides were prepared in a solid phase synthesis using a peptide synthesizer, purified by preparative LC, and characterized using an analytical HPLC system (Shimadzu, Japan) comprised of a C18 column and a MALDI-TOF mass spectrometer (Applied Biosystems, Foster City, CA). The SV40 large T antigen peptide (Pro-Lys-Lys-Arg-Lys-ValCys) was also prepared in the same manner. The protamine sulfate was purchased from Sigma (St. Louis, Missouri). Purified peptides were re-suspended at a concentration of $10 \mu \mathrm{g} / \mu \mathrm{l}$ in $\mathrm{ddH}_{2} \mathrm{O}$ and kept at $-70^{\circ} \mathrm{C}$ prior to further use.

Formation of DPL (DNA/peptide/lipid) and transfection. Plasmid DNA was first mixed with the Tat peptide, and with cationic lipids at various ratios, to obtain the highest possible transfection efficiency. The mixing order of the three components of DPL was examined for optimal transfection. DPL and conventional DNA/lipid (DL) complexes were formed in the TOM transfection media (WelGENE) or PBS. For the formation of DPL, DNA $(0.3 \mu \mathrm{g} / 50 \mu \mathrm{l})$ was mixed with different amounts of the peptide and cationic lipids. The combinations were first formed between those shown in parentheses, and then with the third component: i) (DNA+ peptide)+lipids, ii) (lipids+peptide)+DNA, and iii) (DNA+ lipids)+peptide. Among the 3 components of DPL, one component was re-suspended in $50 \mu 1 \mathrm{TOM}$ media, and then added to the second one in $50 \mu 1$ TOM media. After a 10 -min incubation at room temperature, $50 \mu 1 \mathrm{TOM}$ media containing

Table I. List of peptides.

\begin{tabular}{llc}
\hline Name & \multicolumn{1}{c}{ Peptide sequences } & Size \\
\hline Tat & Arg-Lys-Lys-Arg-Arg-Gln-Arg-Arg-Arg-Pro-Pro-Gln & 12 \\
C-Tat & Cys-Arg-Lys-Lys-Arg-Arg-Gln-Arg-Arg-Arg-Pro-Pro-Gln & 13 \\
Tat-C & Arg-Lys-Lys-Arg-Arg-Gln-Arg-Arg-Arg-Pro-Pro-Gln-Cys & 13 \\
G-Tat & Gly-Arg-Lys-Lys-Arg-Arg-Gln-Arg-Arg-Arg-Pro-Pro-Gln & 13 \\
Tat-G & Arg-Lys-Lys-Arg-Arg-Gln-Arg-Arg-Arg-Pro-Pro-Gln-Gly & 13 \\
Tat-P & Arg-Lys-Lys-Arg-Arg-Gln-Arg-Arg-Arg-Pro-Pro-Gln-Pro & 13 \\
SVp & Pro-Lys-Lys-Arg-Lys-Val-Cys & 7 \\
\hline
\end{tabular}


the third component was added and incubated for a further 10 min. The cationic lipids used were: Lipofectin ${ }^{\mathrm{TM}}$ (LF: DOTMA/DOPE), Oligofectin ${ }^{\mathrm{TM}}$ (OF), DMRIE-C ${ }^{\mathrm{TM}}$ (DMC), Lipofectamine (LFA: DOSPA/DOPE), Lipofectamine plus ${ }^{\mathrm{TM}}$ (LFAP), Lipofectamine ${ }^{\mathrm{TM}} 2000$ (LFAMi) (Invitrogen) and DOTAP (DOTAP/DOPE). Transfections were performed for $5 \mathrm{~h}$ at $37^{\circ} \mathrm{C}$. The transfectants were then added with $150 \mu \mathrm{l}$ TOM media containing $20 \%$ FBS, and incubated for a further $16 \mathrm{~h}$.

Measurement of reporter gene activity after transfection. The luciferase activity of the cells transfected with the luciferase gene was measured using a Luciferase assay kit (Promega, Madison, WI, USA). The transfectants were removed from the culture media, washed twice with PBS (without $\mathrm{Mg}^{2+}$ and $\mathrm{Ca}^{2+}$ ), lysed in $100 \mu \mathrm{l} /$ well 1X CCLR solution [cell culture lysis reagent, $25 \mathrm{mM}$ Tris-phosphate (pH 7.8), 2 mM DTT, 2 mM 1,2-diaminocyclohexane-N,N,N'-tetraacetic acid, 10\% glycerol and $1 \%$ Triton X-100]. The supernatant from the cell lysate was transferred to a 5-ml round bottom test tube and $20 \mu 1$ luciferase assay reagent was added. The luciferase activity was measured for $10 \mathrm{sec}$ in a luminometer (Berthold Detection Systems, Pforzheim, Germany). To measure Bgalactosidase activity, cells transfected with the LacZ plasmid were washed twice with PBS, fixed in $0.05 \%$ glutaraldehyde for $10 \mathrm{~min}$ and incubated in $200 \mu \mathrm{l} /$ well X-gal solution $(0.1 \mathrm{M}$ sodium phosphate, $1 \mathrm{mM}$ magnesium chloride, $150 \mathrm{mM}$ sodium chloride, $1.5 \mathrm{mM}$ potassium ferricyanide, $1.5 \mathrm{mM}$ potassium ferrocyanide and $0.1 \% \mathrm{X}$ gal) for $4 \mathrm{~h}$ in a $\mathrm{CO}_{2}$ incubator at $37^{\circ} \mathrm{C}$. The cells were then subjected to microscopic observation.

In vivo transfection of ribbon antisense oligos by DPL. Antisense and mismatch oligo sequences to human k-ras are as follows: k-ras-RiAS sequence, 5'-GATCCAGGGTTT ACATAATTACACACTTTGTCTTTGACTTCTTTTTC TTCCCTG-3' and k-ras SC scrambled sequence, 5'-GA TCCAGGGATTTCTATTATGTCTTCTGTTTCATTT ACTCTTCATTAACCCTG-3'. Both k-ras antisense and k-ras scrambled sequence (SC) are designed to form a stem-loop structure, placing the antisense sequence in the loop (27). k-ras-RiAS oligos with FITC labeling were synthesized by the incorporation of fluorescein-11dUTP instead of TTP. To make a single-stranded ribbon antisense molecule, the stemloop k-ras AS oligos were ligated overnight at $16^{\circ} \mathrm{C}$ with T4 DNA ligase. The ribbon antisense was purged of unligated linear molecules with exonuclease III treatment.

SW480 cells $\left(1 \times 10^{7}\right.$ cells $)$ in $200 \mu 1$ PBS were injected subcutaneously into the right flanks of 6- to 8-week-old male $\mathrm{BALBc}$ (nu/nu) nude mice (six mice per group). Animals were monitored regularly for tumor occurrence, size and weight. Tumor growth was monitored with a caliper on alternate days. When the desired tumor size was reached (40$50 \mathrm{~mm}^{3}$ ), animals were injected intratumorally with either PBS alone, k-ras-RiAS (100 $\mu \mathrm{g} / \mathrm{mouse})$, or k-ras SC scrambled oligos (100 $\mu \mathrm{g} / \mathrm{mouse})$. The k-ras-RiAS oligos were mixed with the Tat-C peptide and cationic lipids (DOTAP/DOPE) at a ratio of 1:2:2 and administered 6 times on alternate days. Tumors were removed $16 \mathrm{~h}$ after the antisense injection, and were embedded in a Tissue-Tek ${ }^{\mathrm{TM}}$ OCT compound (Miles,
A

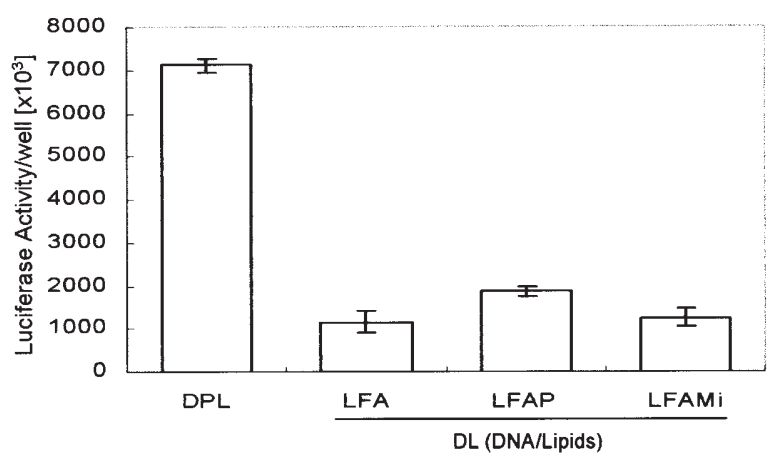

B

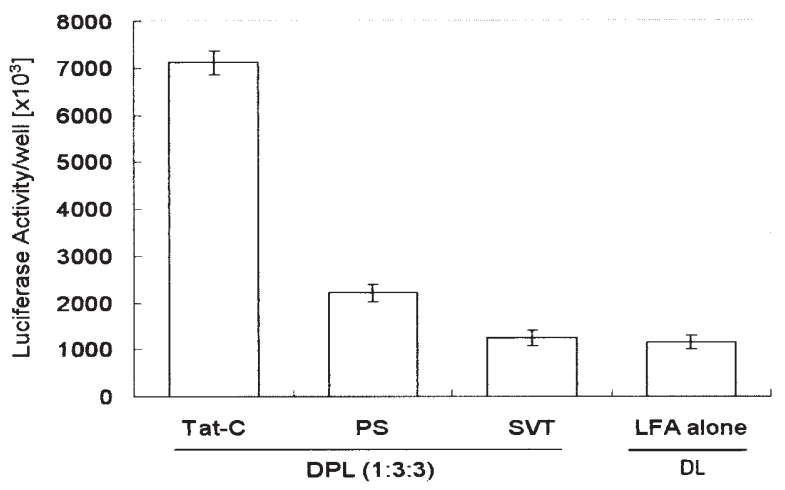

C

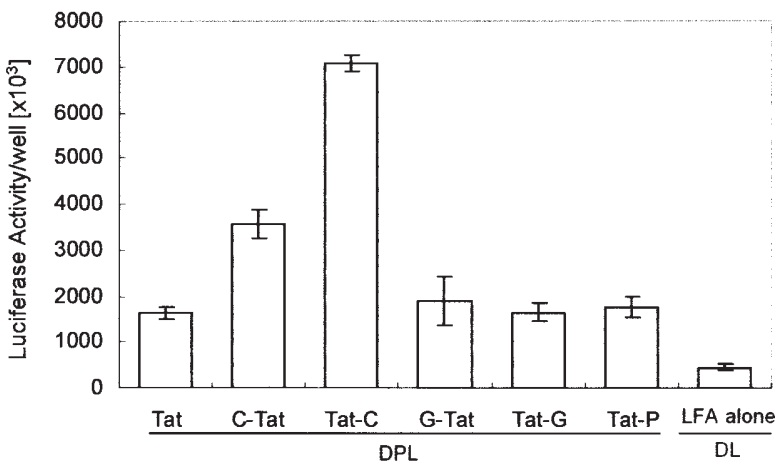

Figure 1. Enhanced transfection achieved using the DPL (DNA/peptide/ lipid) complex in K562 cells. Cells $\left(2 \times 10^{5}\right.$ cells/well) were transfected with DPL and measured for luciferase activity. Plasmid DNA $(0.3 \mu \mathrm{g}$ in $50 \mu \mathrm{l}$ TOM media) was mixed with peptides and cationic lipids. (A) DPL formed with the Tat-C peptide and LFA were compared for transfection efficiency to DL (DNA/lipids). The cationic lipids used were Lipofectamine (LFA), Lipofectamine plus (LFAP) and Lipofectamine 2000 (LFAMi). (B) Various peptides with C-terminal cysteine were used to form DPL and compared for transfection efficiency. A control experiment was performed with the DL transfection using LFA. Tat-C, Tat peptide with a C-terminal cysteine; PS, protamine sulfate with a C-terminal cysteine; and SVT, SV40 large T antigen with a C-terminal cysteine. (C) The Tat peptide was modified at either the $\mathrm{N}$ - or $\mathrm{C}$-terminus by the addition of an amino acid residue. Tat, Tat NLS peptide; C-Tat, Tat peptide with an N-terminal cysteine; G-Tat, Tat peptide with an N-terminal glycine; Tat-G, Tat peptide with a C-terminal glycine; and Tat-P, Tat peptide with a C-terminal proline.

Elkhart, IL, USA) under liquid nitrogen. Tissue blocks of the tumors were cryo-sectioned to $10-\mu \mathrm{m}$ thicknesses and mounted on Poly-Prep ${ }^{\text {тм }}$ slides (Sigma, St. Louis, MO, USA). The tissues were mounted with Synthetic Mountant ${ }^{\mathrm{TM}}$ (Shandon, Pittsburgh, PA, USA) for microscopic observation. The efficacy of FITC-labeled k-ras-RiAS transfer was evaluated in the frozen sections of the tumors using a fluorescent microscope. 


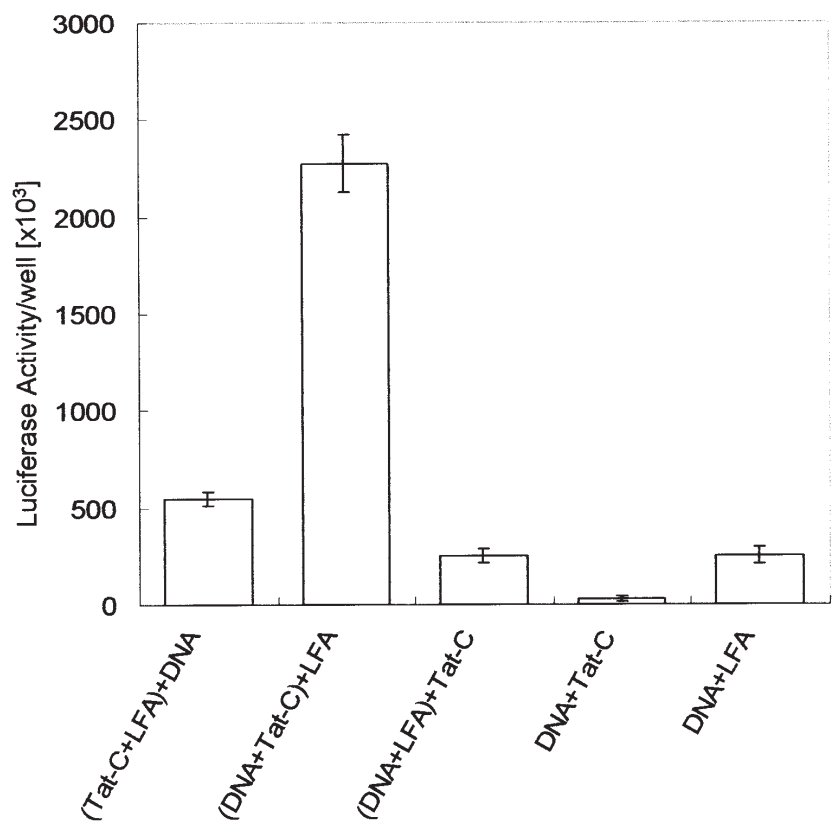

Figure 2. The effect of the mixing order of the DPL components on the transfection efficiency. The transfection complex was first formed with the two components shown in parentheses, and then with the third component shown outside the parentheses. Lane 1, (peptide+lipids)+DNA; lane 2, (DNA+peptide)+lipids; lane 3, (DNA+lipids)+peptide; lane 4, DNA+ peptide; and lane 5, DNA+lipids.

\section{Results}

A modified HIV-1 Tat peptide for the enhanced transfection when forming a DPL (DNA/peptide/lipid) transfection complex. A peptide derived from the HIV-1 Tat protein was previously shown to improve the cellular uptake of macromolecules upon chemical conjugation, or when expressed as a part of recombinant proteins (28-30). The Tat peptide was initially synthesized with a C-terminal cysteine modification for chemical conjugation to cargo DNA molecules for improved transfection. Transfection enhancement has been reported with chemical conjugates of a peptide to oligonucleotides $(31,32)$. In one series of the experiments, the Tat peptide with the C-terminal modification was simply combined with DNA and then with cationic lipids, and compared for transfection efficiency as a control. The tripartite transfection complex was termed DPL (DNA/peptide/lipid) and the conventional DNA/lipid complex as DL (DNA/lipid). To our surprise, the DPL transfection complex resulted in a much enhanced transgene expression than the DL complexes, measured by luciferase expression (Fig. 1A).

Peptides with similar properties, such as the SV40 large T antigen peptide (SVT), and protamine sulfate (PS), harboring nuclear localization signal sequences, were then tested to examine if other peptides were similarly enhancing transfection. When tested for transfection efficiency, the Tat peptide showed $\sim 3$ - and 4-fold enhanced transfection, respectively, compared to those obtained using PS and SVT (Fig. 1B).

As the Tat peptide with the C-terminal cysteine (Tat-C ) exhibited significantly enhanced transfection, the cysteine modification at the $\mathrm{C}$-terminus was studied for its role in the
A

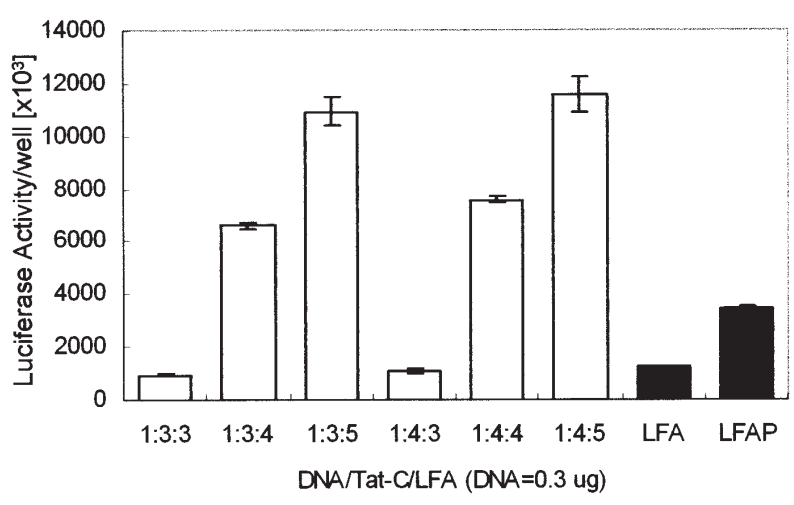

$\mathrm{B}$

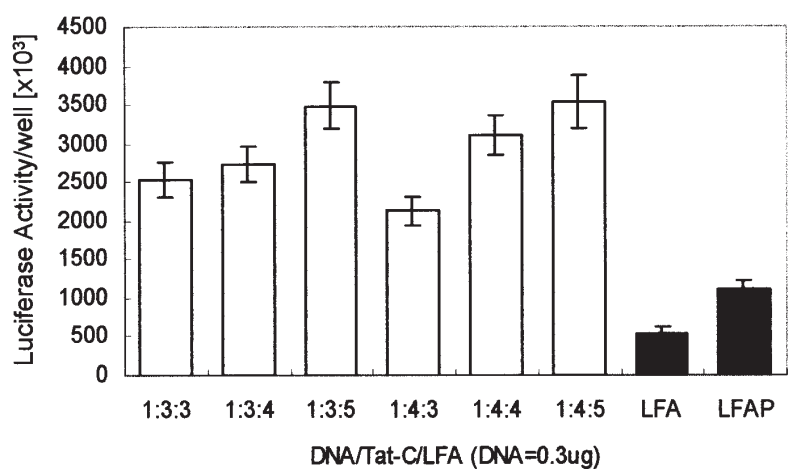

Figure 3. Optimization of the component ratios of DPL. The DPL complex was formed by mixing DNA with different amounts of Tat-C, and then with LFA. Cells $\left(2 \times 10^{5}\right.$ cells/well) were seeded one day prior to transfection, and transfected with DPL at the indicated ratios. The luciferase activity was measured two days after transfection in K562 (A) and HeLa (B) cells.

enhanced transfection of the DPL complex. When the Tat peptide lacking the $\mathrm{C}$-terminal cysteine was used, the peptide showed $\sim 3.5$-fold improved transfection. Similar levels of transfection enhancement were observed when the C-terminus of the Tat peptide was replaced with the other amino acid residues, glycine and proline. Meanwhile, the Tat peptide with an $\mathrm{N}$-terminal cysteine showed $\sim 7$-fold transfection enhancement compared with the DL transfection (Table I; Fig. 1C). With Tat-C, the DPL complex showed $>10$-fold enhancement in transfection. These results demonstrate that the Tat peptide with the C-terminal cysteine is important for enhanced transfection when used in the DPL complex.

Effect of the mixing order and ratio of DPL components on transfection efficiency. Since each component of DPL is a charged molecule, the mixing order of each component may affect the complex formation, thus transfection efficiency. To test this possibility, each component of the transfection complex was mixed in different orders and compared to the transfection efficiency obtained from DL. When Tat-C was first mixed with cationic lipids, and then with DNA [(peptide+lipids)+DNA], the transfection increased 2-fold. When plasmid DNA was mixed with cationic lipids, and then with Tat-C [(DNA+lipids)+peptide], the level of transfection was only comparable to that of DL. Tat-C alone failed to improve the transfection. In contrast, when plasmid DNA was first mixed with the peptide and then with lipids [(DNA+peptide)+lipids; DPL] at a ratio of 1:2:5 (w:w:w), the 


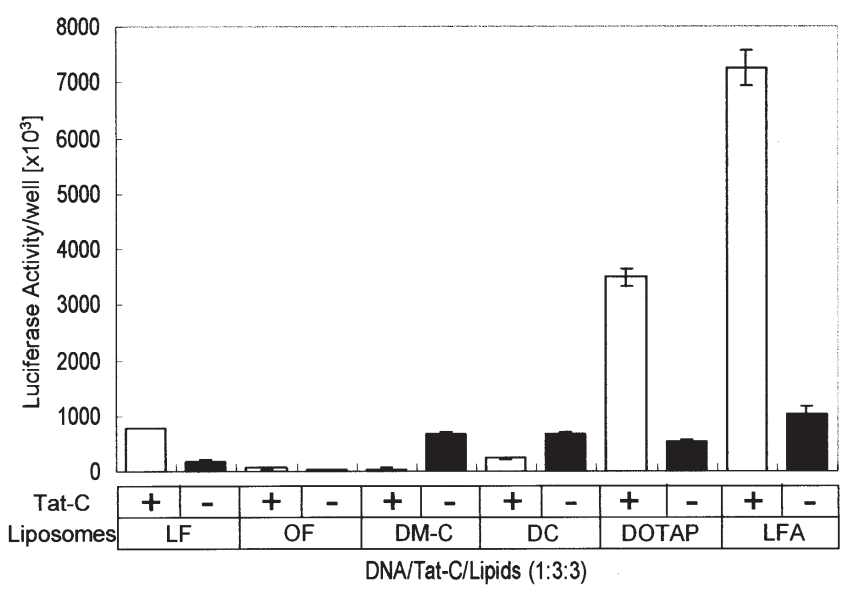

Figure 4. Disparate transfection efficiencies obtained with various lipids. K562 cells $\left(2 \times 10^{5}\right.$ cells/well) were transfected with DPL containing different cationic lipids. Plasmid DNA was mixed with Tat-C, and then with cationic lipids, at the DPL ratio of 1:2:5 (w:w:w). LF, Lipofectin; OF, Oligofectin; DM-C, DMRIE-C; DC, DOTAP-cholesterol; DOTAP, DOTAP-DOPE; and LFA, Lipofectamine. transfection increased by $>9$-fold, as shown by luciferase activity (Fig. 2), demonstrating the importance of a correct mixing order for the formation of an effective DPL complex.

Transfection efficiency was then studied by changing the ratio of each component of the DPL complex. The ratio of each component was varied by using different amounts of peptides and lipids, while maintaining the amount of plasmid DNA constant. The DPL complexes formed with different component ratios were introduced into both HeLa and K562 cells, and examined for luciferase activity. For $0.3 \mu \mathrm{g}$ plasmid DNA, the amounts of peptide and lipids were increased fold-wise, in a weight by weight fashion. When the amount of the Tat-C peptide was increased by $>3$-fold, and the lipids $>5$-fold to the amount of plasmid DNA, the luciferase activity became optimally high (Fig. 3A and B). The transfection efficiencies obtained with a DPL ratio of 1:3:5 in K562 cells were enhanced by $>12$ - and 4-fold compared to those of the respective DL complexes containing either Lipofectamine (LFA) or Lipofectamine plus (LFAP) (Fig. 3A). In HeLa cells, the DPL ratio of 1:3:5 induced a 7-
A

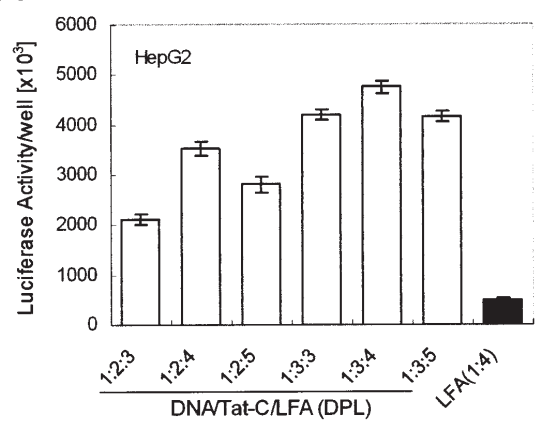

C

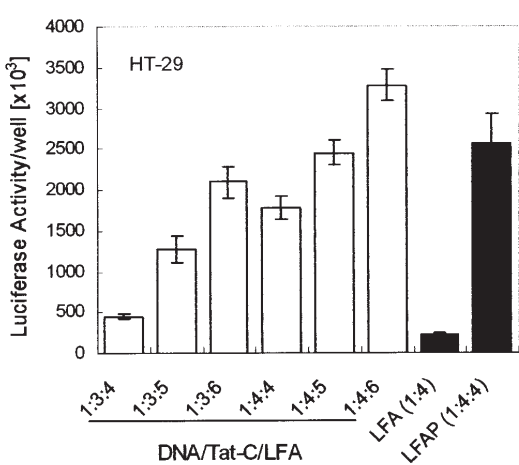

E

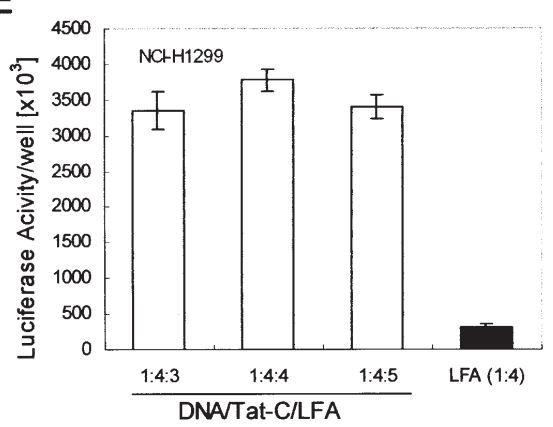

B

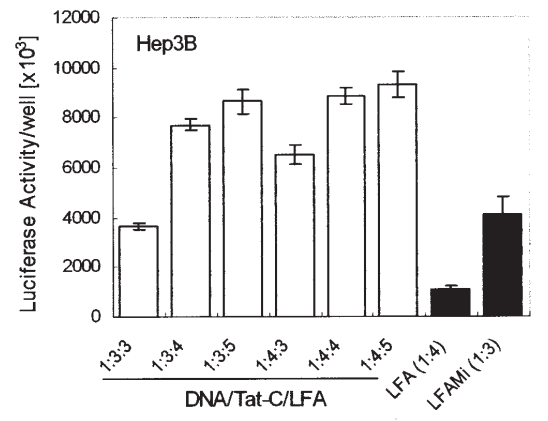

D

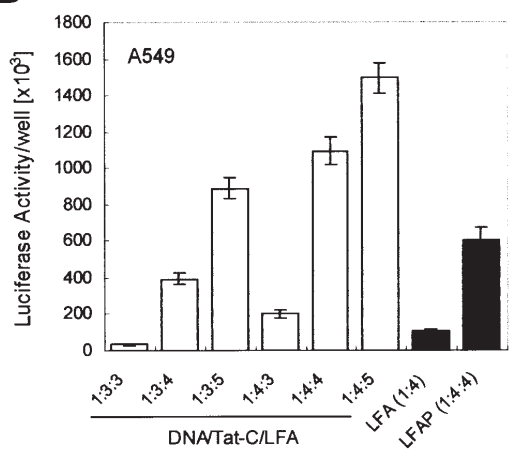

$\mathrm{F}$

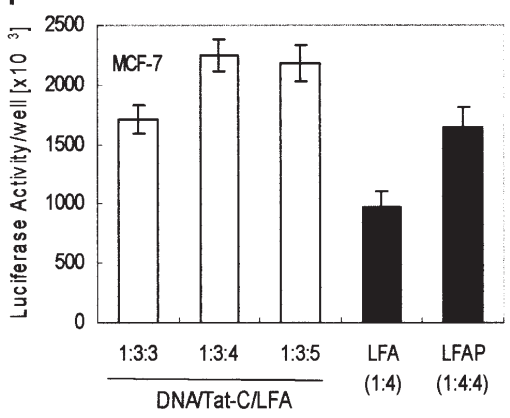

Figure 5. Transfection efficiency in various cell lines using DPL. Cells were seeded at a density of $2 \times 10^{5}$ cells/well for the adherent cells and $5 \times 10^{5}$ cells/well for the suspended cells. Cells were subjected to DPL transfection at the various component ratios, and the luciferase activities were measured two days after transfection. The cationic lipids forming DPL were as follows: LFA, Lipofectamine; LFAP, Lipofectamine plus; and LFAMi, Lipofectamine 2000. Transfected cell lines: (A) HepG2, (B) Hep3B, (C) HT-29, (D) A549, (E) NCI-H1299, and (F) MCF-7. 

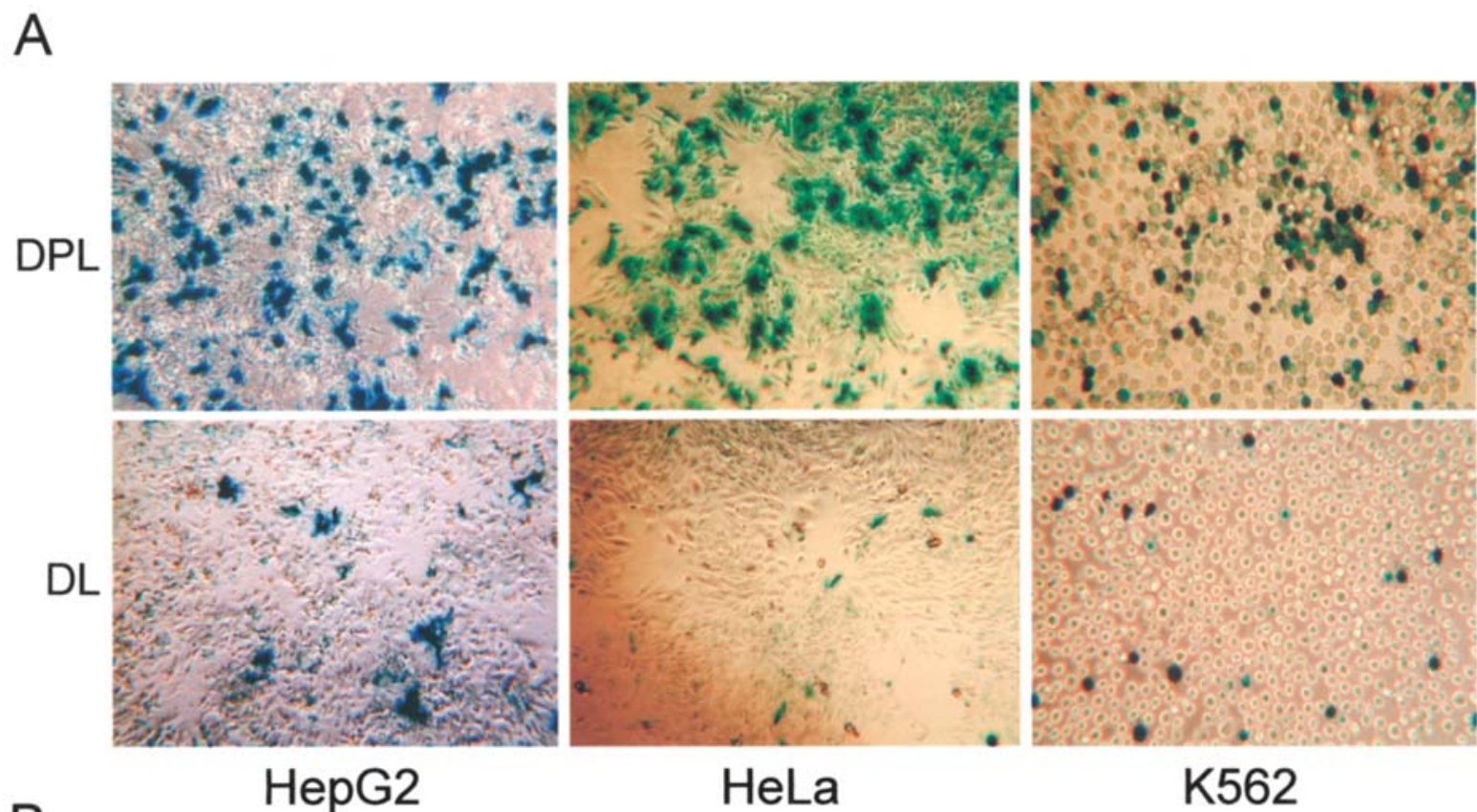

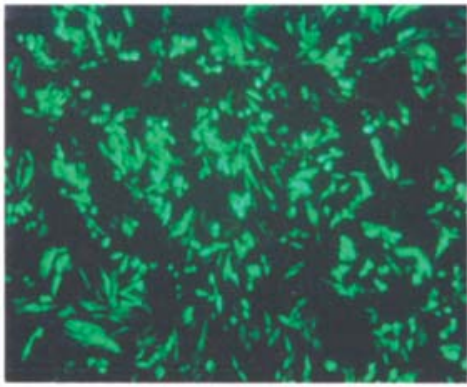

DPL

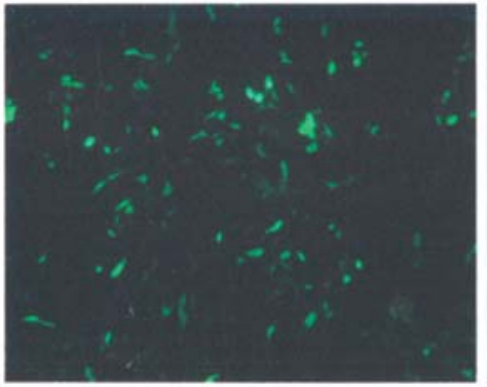

DL

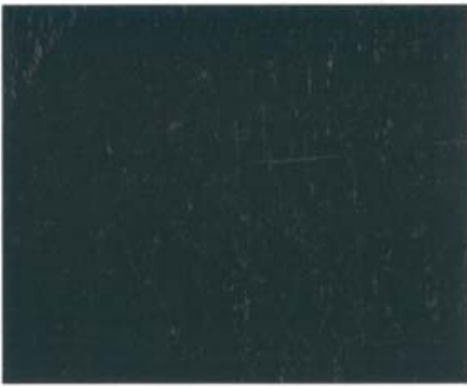

DNA only

Figure 6. Transfection efficiencies of DPL in three cell lines. (A) Cells were transfected with plasmids containing the LacZ gene. The transfections were performed with DPL. (B) Cells were transfected with plasmids containing the GFP gene and were examined under a microscope (x100).

fold increase in transfection compared to LFA alone (Fig. 3B).

Effects of different lipids in DPL on transfection efficiency. The fact that transfection enhancement provided by DPL requires not only the Tat- $\mathrm{C}$ peptide, but also the cationic lipids, prompted us to examine the effect of different cationic lipids on the transfection efficiency. The cationic lipids used for DPL complex formations were Lipofectin (LF: DOTMA/ DOPE), Oligofectin (OF), DMRIE-C (DM-C), DOTAPcholesterol (DC), DOTAP/DOPE (DOTAP) and Lipofectamine (LFA: DOSPA/DOPE). All the lipids were mixed at the DPL ratio of 1:3:5 and examined for luciferase activity in K562 cells. When formulated with DOTAP and LFA, the luciferase activities were found to be 7- and 8-fold higher than those achieved with the respective DL complex. In contrast, LF, OF, DM-C and DC failed to increase transfection efficiency when forming DPL over those obtained from each DL complex with the respective lipids. In fact, LF, DM-C and DC resulted in reduced transfection when combined with Tat-C. These results show that the chemical composition of the different cationic lipids may play an important role in forming an effective DPL (Fig. 4).
Transfection efficiency of DPL in different cell lines. DPL was next tested for transfection enhancement in 6 other cell lines; HepG2, Hep3B, HT-29, A549, NCI-H1299 and MCF-7. The transfection efficiencies were measured for the activities of luciferase, B-galactosidase or GFP (Green fluorescent protein). The optimal ratios of DPL were found to vary in the different cell lines. In the 6 cell lines studied, DPL resulted in much enhanced, but varying degrees of, luciferase activity over those of DL (shown in LFA-mediated transfection), showing 2- to 18 -fold increases in transgene expression (Fig. 5A-F). Similar levels of transfection enhancement were observed for expression of $\beta$-galactosidase and GFP in HepG2, HeLa and K562 cells (Fig. 6A and B).

Effect of serum on the transfection of DPL. Cationic lipids tend to exhibit reduced transfection in the presence of serum (1-4). Transfection mediated by DPL was then tested for the adverse effect on transfection by serum. DPL containing either LFA or DOTAP (DOTAP/DOPE) was employed for transfection either in the presence or absence of serum. The luciferase activity after DPL transfection with LFA was reduced $76 \%$ in the presence of serum, even though the transfection level was still $\sim 2$-fold higher than that of DL 
A

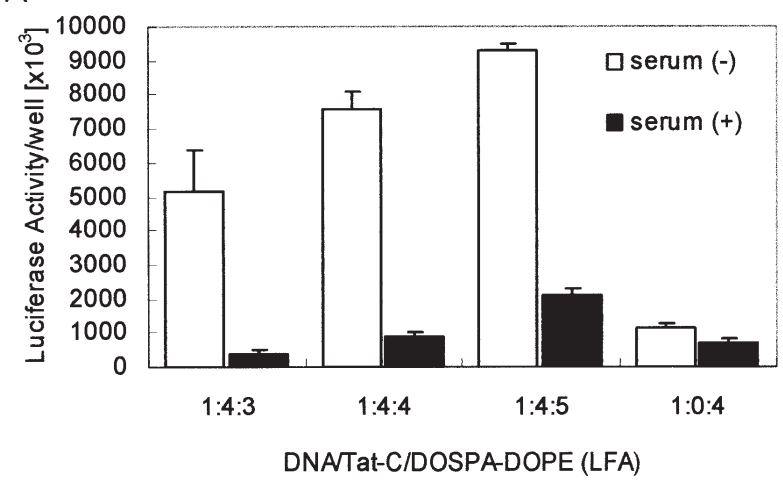

B

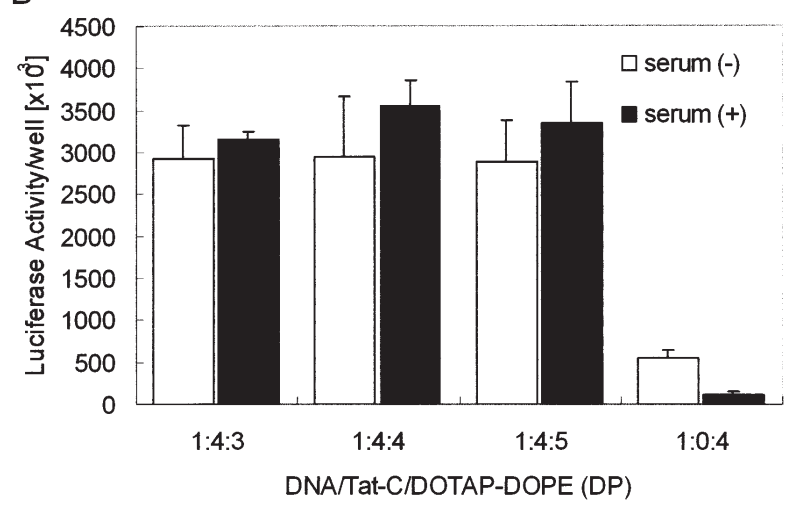

Figure 7. The luciferase activity showing the effect of serum on transfection efficiency. DPL transfections in HeLa cells were performed either in the presence or absence of serum. (A) DPL was formed with LFA. (B) DPL formed with DOTAP. $\square 0 \%$ fetal bovine serum (FBS); $\mathbf{1 0 \%}$ FBS.

transfection with LFA (Fig. 7A). Interestingly, when DOTAP was used, DPL showed a higher transfection level in the presence of serum than in the absence of serum indicating the potential utility of DPL with DOTAP in animal studies (Fig. 7B).

Efficient in vivo tissue uptake of ribbon antisense oligos in $D P L$. We have developed a series of antisense molecules with enhanced stability and low toxicity $(27,33)$. Among these, ribbon antisense (RiAS) is the latest and has been shown to exhibit effective antisense activity with exceptional stability. Successful antisense activity is also heavily dependent on the efficient cellular uptake of antisense molecules (34).

DPL formed with DOTAP was employed for the efficient uptake of antisense oligos in animals. Tumors were formed in nude mice by the subcutaneous inoculation of SW480 that easily forms tumors in mice. Ribbon antisense oligos $(10 \mu \mathrm{g}$ antisense oligos with FITC labeling) to the k-ras mRNA (k-ras-RiAS) were used to form the DPL complex at a ratio of 1:2:2. Tumors were injected intratumorally with DPL when they reached $40-50 \mathrm{~mm}^{3}$. Tumors were harvested $16 \mathrm{~h}$ after the k-ras-RiAS treatment, and were examined for tissue uptake of k-ras-RiAS, detected by fluorescent signals. Whereas DL was not effective in antisense uptake (Fig. 8A, b), k-ras-RiAS in DPL showed strong fluorescent signals in SW480 xenograft tissue (Fig. 8A, c). The antisense activity of k-ras-RiAS in DPL was then examined for its deterrence of tumor growth in animals. Whereas k-ras-RiAS in DPL was able to halt tumor growth to completion, control treatments with naive and mismatched oligos failed to block tumor growth resulting in the increase of tumor mass by $\sim 300 \%$ in 3 weeks (Fig. 8B).

\section{Discussion}

In the present study, the transfection complex of DNA/ peptide/lipid (DPL) was tested for enhanced cellular uptake of plasmid DNA and antisense oligos. Cationic lipids improve cellular uptake of plasmid DNA and antisense oligos, but often result in insufficient and inconsistent cellular uptake. Improved transfection should benefit various cell-based assays as well as gene therapy.

A high level of transfection was achieved when a peptide derived from the nuclear localization signal (NLS) of Tat was added to plasmid DNA prior to the formation of the transfection complex with cationic lipids. The Tat peptide has been used to form chemical conjugates with macromolecules, or expressed as a part of a recombinant protein for enhanced cellular uptake (35). The DPL complex formed by the simple mixing of the three components is an advantage over the use of chemical conjugates for easy preparation of a transfection complex.

The Tat peptide consists largely of basic amino acids, and is derived from the NLS domain of the HIV Tat protein, which has been shown to be capable of intracellular trafficking of small and macromolecules and of crossing the blood brain barrier $(17,36)$. The Tat peptide was shown to be superior to other peptides of similar properties, such as the SV40 large $\mathrm{T}$ antigen peptide and protamine sulfate. This observation shows the importance of the Tat peptide sequence for enhanced cellular uptake. The cysteine residue added to the C-terminus of the Tat peptide is critical for high level transfection, but the underlying mechanism for the enhanced transfection by the cysteine residue merits further investigation.

An ordered mixing and an optimized ratio of each component of DPL were important for enhanced transfection, which suggests that plasmid DNA needs to be pre-condensed by the Tat peptide. The DNA/peptide complex formed is then bound to cationic lipids to form an effective transfection complex. Having an optimal ratio of each component is important in attaining the desired structure and/or net charge of the transfection complex.

Six different cationic lipids were shown to have varying degrees of transfection efficiency upon forming DPL. Among the lipids tested, the transfection mediated by DOSPA/DOPE was highest, followed by DOTAP/DOPE. In contrast, the transfection was not improved by the inclusion of the other lipids. Different cationic lipids exhibited markedly disparate cellular uptake of DPL, indicating that minor charges and/or structural differences are critical to form an effective DPL complex.

DPL was effective to varying degrees in different cell lines. Thus, fine adjustment of the component ratio of DPL may be needed to obtain optimal transfection for each cell type. DPL consists of plasmid DNA of an anionic charge, the Tat peptide of basic amino acids and cationic lipids. The 

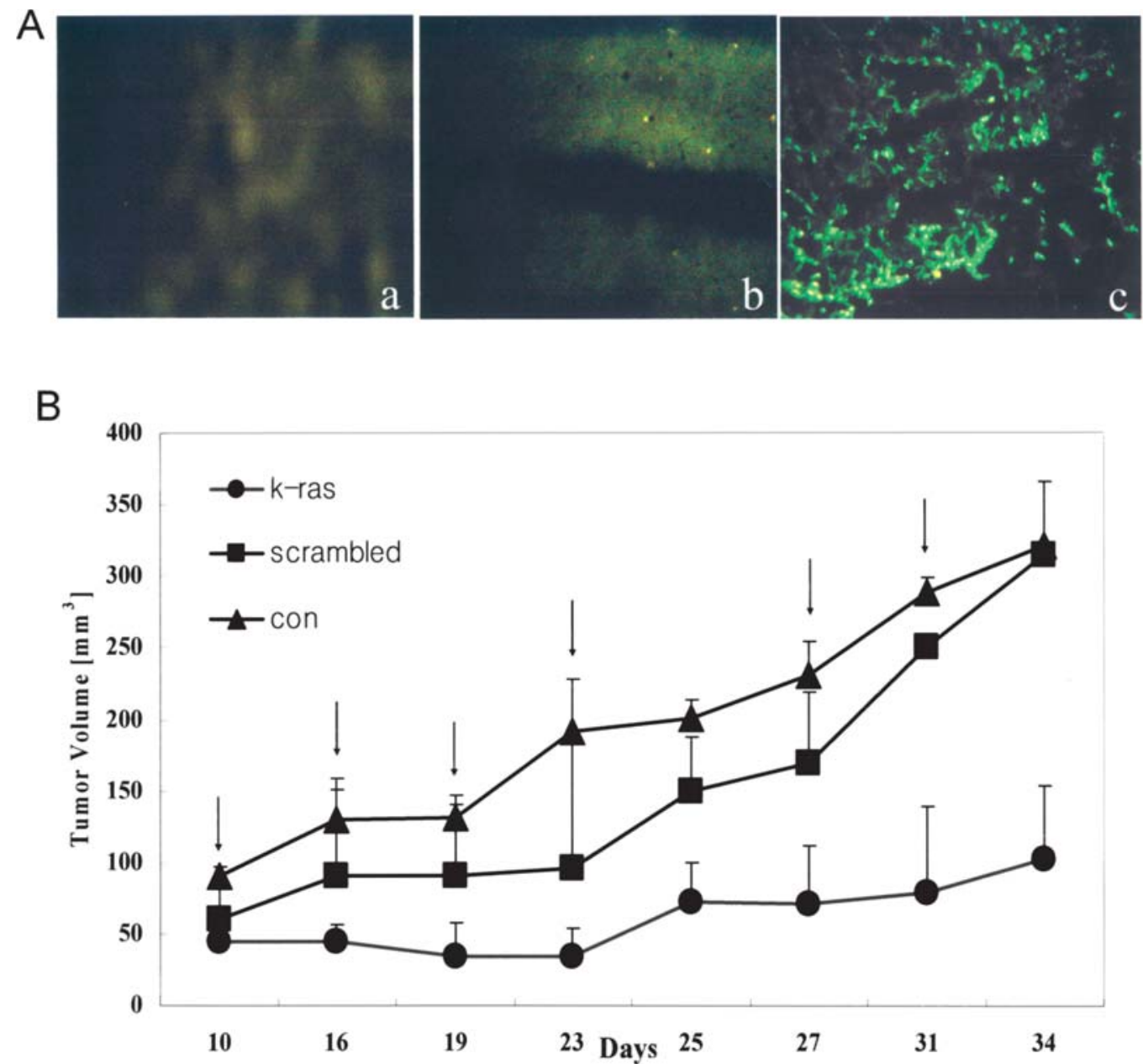

Figure 8. Efficient tissue uptake of ribbon antisense oligos by DPL. Tumors were formed by the subcutaneous injection of SW480. The k-ras-RiAS oligos with FITC labeling were mixed with the Tat-C peptide and cationic lipids (DOTAP/DOPE) at the ratio of 1:2:2 (w:w:w). (A) Tumor tissues were harvested $16 \mathrm{~h}$ after a single injection of DPL. Perfusion-fixed tumor tissue blocks were cryo-sectioned, and the tissues were mounted with the Synthetic Mountant ${ }^{\mathrm{TM}}$ for microscopic observation (magnification, x200). a, FITC-RiAS alone (D); b, FITC-RiAS+cationic lipid (DL); c, FITC-RiAS+Tat-C+cationic lipid (DPL). (B) Effective size reduction of the tumor tissue by DPL transfection of k-ras-RiAS in nude mice. DPL was administered intratumorally 6 times on alternate days as denoted by arrows. k-ras, (k-ras-RiAS) ribbon antisense oligos to the k-ras mRNA; scrambled, (k-ras SC) scrambled ribbon oligos to k-ras; con, naive injection with PBS alone.

complex is formed by electrostatic charge interactions that appear to be affected by the environmental $\mathrm{pH}$ during their formation. The fact that optimal transfection was obtained by DPL formed at a neutral $\mathrm{pH}$, but not in acidic and basic solutions (data not shown), supports the importance of the charge to charge interactions of each component.

In general, antisense oligos show poor cellular uptake due to anionic charges on the molecule's polymeric backbone. Cellular uptake of antisense oligos can be enhanced by forming complexes with cationic lipids (37). Although cationic lipids have several advantages such as low toxicity and simple production, transfection efficiency needs further improvement. It has been shown that a protein fused with the protein transduction domain of the tat gene of the human immunodeficiency virus (HIV) can be efficiently delivered to most tissues in mice (17). The Tat peptide, residues from 4957 , covalently harnessed on the surface of liposomes increases intracellular delivery of bound DNA (20). DPL was able to significantly enhance the cellular uptake of ASoligos, often resulting in $>90 \%$ cells positive for AS-oligo uptake. Improved antisense activity in animals shown in this report may be, in part, explained by the enhanced cellular uptake of antisense oligos.

The DPL complex has also been shown to be very effective for the transfection of plasmid DNA, and is much easier to formulate than are chemical conjugates that are formed with either lipids or proteins. DPL, although providing much enhanced transfection, needs future improvement in cells refractory to transfection and in the ability of tissue-specific targeting.

\section{Acknowledgements}

This study was supported by a generous grant of the CDRC of Korean Science and Engineering Foundation (R13-2002028-010040-0).

\section{References}

1. Pouton CW and Seymour LW: Key issues in non-viral gene delivery. Adv Drug Deliv Rev 46: 187-203, 2001.

2. Akhtar S, Hughes MD, Khan A, Bibby M, Hussain M, Nawaz Q, Double J and Sayyed P: The delivery of antisense therapeutics. Adv Drug Deliv Rev 44: 3-21, 2000. 
3. Lebedeva I, Beimetskaya L, Stein CA and Vilenchik M: Cellular delivery of antisense oligonucleotides. Eur J Pharm Biopharm 50: 101-119, 2000.

4. Ghaumont CG, Seksek O, Grzybowska J, Borowski E and Bolard J: Delivery systems for antisense oligonucleotides. Pharmacol Ther 87: 255-277, 2000.

5. Kataoka K and Harashima H: Gene delivery systems: viral vs. non-viral vectors. Adv Drug Deliv Rev 52: 151, 2001.

6. Wang II and Huang II: Adenovirus technology for gene manipulation and functional studies. Drug Discov Today 5: 10-16, 2000.

7. Luo D and Salzman WM: Synthetic DNA delivery systems. Nat Biotechnol 18: 33-37, 2000.

8. Brokx R and Gariepy J: Peptide- and polymer-based gene delivery vehicles. Methods Mol Med 90: 139-160, 2004.

9. Jordan M and Wurm F: Transfection of adherent and suspended cells by calcium phosphate. Methods 33: 136-143, 2004.

10. Banga AK and Prausnitz MR: Assessing the potential of skin electroporation for the delivery of protein- and gene-based drugs. Trends Biotechnol 16: 408-412, 1998.

11. Fulka J Jr, Loi P, Fulka H, Ptak G and Nagai T: Nucleus transfer in mammals: noninvasive approaches for the preparation of cytoplasts. Trends Biotechnol 22: 279-283, 2004.

12. Washbourne P and McAllister AK: Moving on to the cargo problem of microtubule-dependent motors in neurons. Curr Opin Neurobiol 12: 566-573, 2002.

13. Zelphati O, Uyechi LS, Barron LG and Szoka FC Jr: Effect of serum components on the physico-chemical properties of cationic lipid/oligonucleotide complexes and on their interactions with cells. Biochim Biophys Acta 1390: 119-133, 1998.

14. Morris MC, Chaloin L, Heitz F and Divita G: Translocating peptides and proteins and their use for gene delivery. Curr Opin Biotechnol 11: 461-466, 2000.

15. Schwarz JJ and Zhang S: Peptide-mediated cellular delivery. Curr Opin Mol Ther 2: 162-167, 2000.

16. Vives E, Brodin P and Lebleu B: A truncated HIV-1 Tat protein basic domain rapidly translocates through the plasma membrane and accumulates in the cell nucleus. J Biol Chem 272: 16010-16017, 1997.

17. Schwarze SR, Ho A, Vocero-Akbani A and Dowdy SF: In vivo protein transduction: Delivery of a biologically active protein into the mouse. Science 285: 1569-1572, 1999.

18. Eguchi A, Akuta T, Okuyama H, Senda T, Yokoi H, Inoguchi H, Fujita S, Hayakawa T, Takeda K, Hasegawa M and Nakanishi M: Protein transduction domain of HIV-1 Tat protein promotes efficient delivery of DNA into mammalian cells. J Biol Chem 276: 26204-26210, 2001.

19. Futaki S, Suzuki T, Ohashi W, Yagami T, Tanaka S, Ueda K and Sugiura Y: Arginine-rich peptides. J Biol Chem 276: 5836-5840, 2001.

20. Torchilin VP, Rammohan R, Weissig V and Levchenkol TS: TAT peptide on the surface of liposomes affords their efficient intracellular delivery even at low temperature and in the presence of metabolic inhibitors. Proc Natl Acad Sci USA 98: 8786-8791, 2001

21. Zanta MA, Valladier PB and Behr JP: Gene delivery: a single nuclear localization signal peptide is sufficient to carry DNA to the cell nucleus. Proc Natl Acad Sci USA 96: 91-96, 1999.
22. Ludtke JJ, Zhang G, Sebestyen MG and Wolff JA: A nuclear localization signal can enhance both the nuclear transport and expression of 1 kb DNA. J Cell Sci 112: 2033-2041, 1999.

23. Derossi D, Calvet S, Trembleau A, Brunissen A, Chassaing G and Prochiantz A: Cell internalization of the third helix of the Antennapedia homeoprotein is receptor-independent. J Biol Chem 271: 18188-18193, 1996.

24. Dokka S, Toledo D, Shi X, Ye J and Rojanasakul Y: Highefficiency gene transfection of macrophages by lipoplexes. Int $\mathbf{J}$ Pharm 206: 97-104, 2000.

25. Sorgi FL, Bhattacharya S and Huang L: Protamine sulfate enhances lipid-mediated gene transfer. Gene Therapy 4: 961-968, 1997.

26. Bharath MM, Chandra NR and Rao MR: Prediction of an HMG-box fold in the C-terminal domain of histone $\mathrm{H} 1$ : insights into its role in DNA condensation. Proteins 49: 71-81, 2002.

27. Moon IJ, Choi K, Kim JE, Lee Y, Schreiber AD and Park JG: Potent growth inhibition of leukemic cells by novel ribbon-type antisense oligonucleotides to $c-m y b$. J Biol Chem 275: 4647-4653, 2000.

28. Yang Y, Ma J, Song Z and Wu M: HIV-1 TAT-mediated protein transduction and subcellular localization using novel expression vectors. FEBS Lett 532: 36-44, 2002.

29. Silhol M, Tyagi M, Giacca M, Lebleu B and Vives E: Different mechanisms for cellular internalization of the HIV-1 Tatderived cell penetrating peptide and recombinant proteins fused to Tat. Eur J Biochem 269: 494-501, 2002.

30. Fawell S, Seery J, Daikh Y, Moore C, Chen LL, Pepinsky B and Barsoum J: Tat-mediated delivery of heterologous proteins into cells. Proc Natl Acad Sci USA 91: 664-668, 1994.

31. Turner JJ, Arzumanov AA and Gait MJ: Synthesis, cellular uptake and HIV-1 Tat-dependent transactivation inhibition activity of oligonucleotide analogues disulphide-conjugated to cell-penetrating peptides. Nucleic Acids Res 33: 27-42, 2005.

32. Moulton HM, Nelson MH, Hatlevig SA, Reddy MT and Iversen PL: Cellular uptake of antisense morpholino oligomers conjugated to arginine-rich peptides. Bioconjug Chem 15: 290-299, 2004.

33. Moon IJ, Lee Y, Kwak CS, Lee JH, Choi K, Schreiber AD and Park JG: Target site search and effective inhibition of leukaemic cell growth by a covalently closed multiple anti-sense oligonucleotide to $c-m y b$. Biochem J 346: 295-303, 2000.

34. Matsuda M, Park JG, Hunter S, Chien P and Schreiber AD: Abrogation of the $\mathrm{Fc}$ gamma receptor IIA-mediated phagocytic signal by stem-loop Syk antisense oligonucleotides. Mol Biol Cell 7: 1095-1106, 1996.

35. Lee HJ and Pardridge WM: HIV-1 Tat-mediated effects on focal adhesion assembly and permeability in brain microvascular endothelial cells. Bioconjug Chem 12: 995-999, 2001.

36. Avraham HK, Jiang $\mathrm{S}$, Lee TH, Prakash $\mathrm{O}$ and Avraham S: HIV-1 Tat-mediated effects on focal adhesion assembly and permeability in brain microvascular endothelial cells. J Immunol 173: 6228-6233, 2004

37. Wheeler CJ, Felgner PL, Tsai YJ, Marshall J, Sukhu L, Doh SG, Hartikka J, Nietupski J, Manthorpe M, Nichols M, Plewe M, Liang X, Norman J, Smith A and Cheng SH: A novel cationic lipid greatly enhances plasmid DNA delivery and expression in mouse lung. Proc Natl Acad Sci USA 93: 11454-11459, 1996. 